\title{
The Global Threat of Bird (Avian) Flu its Treatment Methods and Public Health Preventive Measures
}

\author{
Ahsan Ali Siddiqui* \\ Assistant Professor Family Medicine, Ministry of Health Saudi Arabia, Saudi Arabia
}

Received: 16 October, 2018; Accepted: 5 November, 2018; Published: 15 November, 2018

*Corresponding author: Ahsan Ali Siddiqui, Assistant Professor Family Medicine, Ministry of Health Saudi Arabia, Saudi Arabia,

E-mail: drahsan77@hotmail.com

\begin{abstract}
Background and Objectives: This assignment will critically review and evaluate the mortality and the harmful effects caused by the Bird (Avian) Flu in Thailand and Turkey. Are we prepared for the management and treatment of Bird (Avian) Flu if it affects the UK and other non-affected countries?
\end{abstract}

Methods: Literature Review was done carefully for the Hot Current Topic Avian Flu and what are the Preventive Measures. Various Recommended and Good repute Global Organizations such as WHO, CDC USA, BBC UK, NHS UK Others Literature was reviewed for Article writing and selecting Excellent Preventive Measures $[1,3-7,12,16,18]$.

Results: This essay has explored the Avian (bird) flu in terms of definition, classification, signs and symptoms caused by Avian flu, statistics of morbidity and mortality caused by Avian flu in Turkey, Thailand and other effected countries. In this essay I talk treatment, health implications, risks and the warnings about the Avian flu and food safety implications in humans to prevent from the harmful effects of the Avian flu. I also discuss the some of the actions taken by the UK government to prevent the population of the UK from harmful effects of the Avian flu.

Conclusion: In this essay, I told the dangers and the harmful effects done by the Avian flu and how this deadly disease spread in the communities silently and the health departments comes to know about this disease when it done its work, killing the innocent people, terrorize the whole community by its deadly results. This essay also points out the flaws in our health systems for example, are the health authorities is aware of the dangers of Avian flu are the health departments are serious about giving training to the members of health department to cope with this great threat of Avian flu, are they teaching local people how to cope with this danger? No I don't think that enough work is done to handle this great threat of Avian flu.

Keywords: Avian Flu H5N1; Antiviral Agents; Vaccines; Global Threat

\section{Introduction}

Avian flu (H5N1) is a type of influenza A that mainly infects birds but occasionally affects humans. Avian flu can be transmitted from live birds to people, although transmission between humans is very inefficient. Wild birds are the natural hosts of the virus - hence, the name avian influenza or bird flu [3]. The initial symptoms of avian flu are similar to those of other influenza viruses, including fever, generalised muscle pain, cough and sore throat. However, it is more likely to result in high fever, chest infection, respiratory failure, multi-organ failure, and death [3]. The global threat of Avian flu is getting more attention nowadays as its harmful effects breaks on Turkey resulting in mortality of dozens of people and making hundreds of people sick and killing of thousands of poultry including chickens, ducks and turkeys. This is the most recent outbreak and news heard in the media today in 2006. This essay will discuss the morbidity and the mortality resulting from the Avian (bird) flu in Turkey and Thailand and evaluate whether there is enough preparation for the management and the treatment of the Avian flu if it affected the UK and the countries not affected at the moment. This essay will also explore answers to the following question: Are all the precautions taken against the Avian flu in the UK and other countries? Are the treatment and the medicines effective enough against the Avian flu?

Secretary Mike Leavitt U.S department of health and human services announced additional purchases of antiviral drugs that could be used in the event of a potential influenza pandemic [16]. The department has ordered 2.2 million more treatment courses of antiviral drug zanamivir (Relenza®) from GlaxoSmithKline and 3.8 million more treatment courses of oseltamivir phosphate (Tamiflu ${ }^{\circledR}$ ) from Roche. With these purchases, the Strategic National Stockpile will have a total of 26 million treatment courses of antiviral drugs for distribution to the states when pandemic is deemed to be imminent. By doing stockpiling and making strategies against avian flu pandemic U.S government is doing well for their citizens. USA is now preparing 30 million avian flu vaccines each year for USA citizens. Alaska is the opening door to north America for avian flu because all the migratory birds comes from Asia to America through Alaska so all the health agencies and public health departments are concerned about it and Alaska is the place where all the funds will be utilized this year in 2006.

The European Union (EU) and United Kingdom (UK) controls are in place aimed at preventing the occurrence of bird flu in the UK and elsewhere in the regions. Nonetheless, it remains a remote possibility that bird flu could be introduced to poultry through the migration of wild birds, the illegal importation of dead chickens for consumption, the illegal importation of live birds or the entry 
into the UK of a person who has acquired the illness in an infected area [8]. A French scientist Hoffman developed a technique to reverse engineer viruses, breaking them down into their eight basic components. From there it's a relatively simple process to reassemble the virus, but substitute some of the components and create a vaccine [11]. The UK and EU have to check medically at the airport, the passengers coming from the countries affected by Avian flu. They should check the imports or either banned the imports temporarily if it's necessary.

Infected birds pass on H5N1 through their saliva, nasal secretions, and feces. Other birds may pick up the virus through direct contact with these excretions or when they have contact with surfaces contaminated with this material [17]

The spread of avian influenza viruses from an ill person to another person has been reported very rarely, and transmission has not been observed to continue beyond one person [15]. During an outbreak of avian influenza among poultry, there is a possible risk to people who have direct or close contact with infected birds or with surfaces that have been contaminated with secretions and excretions from infected birds.

\section{The Global Threat of Avian Flu}

Avian (bird) flu is now becoming a great threat to both humans and animals. International health organizations like W.H.O and the group of richest countries for example, G-8 and the government of the effected countries from Avian flu now have to make genuine policies and take serious actions to prevent their innocent population from the harmful effects and deaths caused by Avian flu. United Kingdom have made good policies to cope with Avian flu like doing medical checkups on airports from passengers arriving from the effected countries and not buying the poultry and eggs from the effected countries. A large quantity of vaccines for the emergency treatment of the Avian flu is also prepared by the health authorities in the U.K and stored for the emergency situation. These actions will surely prevent the population of United Kingdom from harmful effects of the Avian flu.

During the late 2003 and early 2004, outbreaks of highly pathogenic avian influenza A (H5N1) occurred among poultry in 8 countries in Asia: Cambodia, China, Indonesia, Japan, Laos, South Korea, Thailand, and Vietnam. At that time, more than 100 million birds either died from the disease or were destroyed in an attempt to prevent further spread of the disease [4-7].

From December 30, 2003 to March 17, 2004, 12 confirmed human cases of avian influenza A (H5N1) were reported in Thailand and 23 in Vietnam, resulting in 23 deaths. By late February 2004, however, the number of new human H5N1 cases being reported in Thailand and Vietnam slowed and then stopped. Within a month, countries in Asia were reporting that the avian influenza outbreak among poultry had been contained [13]

In late June 2004, new outbreaks of lethal avian influenza A (H5N1) infection among poultry were reported by several countries in Asia: Cambodia, China, Indonesia, Malaysia, Thailand, and Vietnam. Since May 2005, outbreaks of H5N1 disease have been reported among poultry in Russia, China, Kazakhstan, Turkey, Romania, and Ukraine [14]. China, Croatia, Mongolia, and Romania also have reported outbreaks of H5N1 in wild, migratory birds since May 2005.

Thailand reported new human cases of H5N1 in October, November, and December 2005, and Vietnam reported new human cases in November 2005. China reported the country's first confirmed human cases in November 2005 and continued to report human cases in December 2005 [3].

The symptoms of avian flu in humans are similar to other strains of the flu and include fever, fatigue, cough, sore throat, eye infections, muscle aches, pneumonia, acute respiratory distress, viral pneumonia, and other severe and life-threatening complications [2].

\section{Treatment \& Anti Viral Agents for Avian Influenza}

Four different influenza antiviral drugs (amantadine, rimantadine, oseltamivir, and zanamivir) are approved by the U.S. Food and Drug Administration (FDA) for the treatment of influenza; three are approved for prophylaxis. All four have activity against influenza A viruses [3]. However, sometimes influenza strains can become resistant to these drugs, and therefore the drugs may not always be effective. There is currently no commercially available vaccine to protect humans against the H5N1 virus that is being detected in Asia and Europe. However, vaccine development efforts are taking place. Research studies to test a vaccine that will protect humans against $\mathrm{H} 5 \mathrm{~N} 1$ virus began in April 2005, and a series of clinical trials is under way.

Two drugs (in the neuraminidase inhibitors class), oseltamivir (commercially known as Tami flu) and zanamivir (commercially known as Relenza) can reduce the severity and duration of illness caused by seasonal influenza. The efficacy of the neuraminidase inhibitors depends, among others, on their early administration (48 hours after symptom onset). For cases of human infection with H5N1, the drugs may improve prospects of survival, if administered early, but clinical data are limited [3]. The drugs now available in market to treat Avian flu if diagnosed in early stage but still a lot of research needed to manufacture more efficient and readily available drugs to combat against the great threat of Avian flu.

\section{Public Health Implications}

The widespread persistence of $\mathrm{H} 5 \mathrm{~N} 1$ in poultry populations poses two main risks for human health.

The first is the risk of direct infection when the virus passes from poultry to humans, resulting in very severe disease. H5N1 has caused the largest number of cases of severe disease and death in humans [3]. Unlike normal seasonal influenza, where infection causes only mild respiratory symptoms in most people, the disease caused by H5N1 follows an unusually aggressive clinical course, with rapid deterioration and high fatality. Primary viral pneumonia and multi-organ failure are common. In the present outbreak, more than half of those infected with the virus have died. 
A second risk, of even greater concern, is that the virus - if given enough opportunities - will change into a form that is highly infectious for humans and spreads easily from person to person. Such a change could mark the start of a global outbreak (a pandemic) [3]. A pandemic can start when three conditions have been met: a new influenza virus subtype emerges; it infects humans, causing serious illness; and it spreads easily and sustainably among humans. The H5N1 virus amply meets the first two conditions: it is a new virus for humans (H5N1 viruses have never circulated widely among people), and it has infected more than 100 humans, killing over half of them. No one will have immunity should an H5N1-like pandemic virus emerge.

Pandemics of flu are due to the emergence of a new flu and are markedly different from recently circulating strains. Few - if any - people will have any immunity to this new virus. This allows it to spread widely, easily and to cause more serious illness. Once a pandemic of influenza starts, everybody will be at risk of catching it. Certain groups may be at greater risk than others: until the virus starts circulating we will not know for sure who the risk groups will be. One case of probable person-to-person spread was recorded among close family members in Thailand. Transmission was limited to one "generation" of spread - that is, it was passed from Person A to Person B, but transmission stopped there. Person B did not spread the disease any further. No additional human-to-human cases have been reported.

The most important warning signal comes when clusters of patients with clinical symptoms of influenza, closely related in time and place, are detected, as this suggests human-to-human transmission is taking place $[18,20-23]$. For similar reasons, the detection of cases in health workers caring for H5N1 patients would suggest human-to-human transmission. Detection of such events should be followed by immediate field investigation of every possible case to confirm the diagnosis, identify the source, and determine whether human-to-human transmission is occurring. Studies of viruses, conducted by specialized WHO reference laboratories, can corroborate field investigations by spotting genetic and other changes in the virus indicative of an improved ability to infect humans. This is why WHO repeatedly asks affected countries to share viruses with the international research community.

No one knows with certainty can a pandemic be prevented The best way to prevent a pandemic would be to eliminate the virus from birds, but it has become increasingly doubtful if this can be achieved within the near future.

Vaccines: Influenza vaccines are the optimal means of preventing influenza. Current influenza vaccines usually protect about 70-90 percent of healthy adults who are vaccinated each year Influenza vaccines are reformulated annually because human-adapted influenza viruses tend to mutate. There are several barriers to preventing avian influenza by vaccination that will need to be overcome. First, preliminary studies suggest that the quantity of $\mathrm{H} 5$ protein needed in each dose to induce a successful immune response may be four or more times that in standard vaccines. Second, two doses may be needed.
Third, the H5 viruses have been mutating. It's unclear whether vaccine produced today will cover potential future strains that are spreading in people. Fourth, normally, it takes about 4-6 months to produce an influenza vaccine. Thus, comprehensive surveillance of influenza, particularly in Asia, is needed to have as much as possible $[9,10]$.

Antiviral drugs: There are two categories of drugs that can treat or prevent influenza, neuraminidase inhibitors (e.g., oseltamivir) and adamantine. Only the neuraminidase inhibitors appear to be effective against the H5 viruses currently in circulation. Expert committees have recommended that a high priority be placed on the use of such drugs for both treatment and prevention in the event of a pandemic [3].

Population-based measures: Other strategies to minimize transmission of influenza may include isolating infected patients, eliminating large gatherings of people such as sporting events, shutting down other places where people congregate such as schools and places of employment, and use of "respiratory etiquette" including covering the mouth while coughing, frequent hand washing, and wearing face masks. Quarantine and isolation are of particularly limited practicality for influenza due to the short incubation period (1-4 days) and ability of an infected individual to transmit virus prior to developing symptoms of illness (up to 24 hours before symptoms) [3]. These three categories of interventions are well known by the health staff and the local communities as these information are available on news and websites but I believe still a lot of research and work is needed to give information and to prevent local people from this great threat of Avian flu.

\section{Food Safety Implications in Humans}

1. Cooking (temperatures at or above $70^{\circ} \mathrm{C}$ in all parts of food item) will inactivate the H5NI virus. Properly cooked poultry meat is therefore safe to consume.

2. The H5N1 virus, if present in poultry meat, is not killed by refrigeration or freezing

3. Home slaughtering and preparation of sick or dead poultry for food is hazardous: this practice must be stopped.

4. Eggs can contain H5N1 virus both on the outside shell and the inside whites and yolk. Eggs from areas with H5N1 outbreaks in poultry should not consumed raw or partially cooked runny yolk. Uncooked eggs should not be used in foods that will not be cooked, baked or heat treated in other ways [19].

5. There is no epidemiological evidence to indicate that people have been infected with the H5N1 virus following consumption of properly cooked poultry or eggs [19].

Experts say, avian flu is not a food-borne virus so eating chicken is safe. The only people thought to be at risk are those involved in the slaughter and preparation of meat that may be infected [1]. However, the WHO recommends, to be absolutely safe all meat should be cooked to a temperature of at least $70^{\circ} \mathrm{C}$. Eggs should also be thoroughly cooked. These are the guidelines provided to local community, are people following these guide 
lines? Governments and their health departments have to check strictly that people are following these to combat the Avian flu.

\section{Conclusion}

This essay has explored the Avian (bird) flu in terms of definition, classification, signs and symptoms caused by Avian flu, statistics of morbidity and mortality caused by Avian flu in Turkey, Thailand and other effected countries. In this essay I talk treatment, health implications, risks and the warnings about the Avian flu and food safety implications in humans to prevent from the harmful effects of the Avian flu. I also discuss the some of the actions taken by the UK government to prevent the population of the UK from harmful effects of the Avian flu. In this essay, I told the dangers and the harmful affects done by the Avian flu and how this deadly disease spread in the communities silently and the health departments comes to know about this disease when it done its work, killing the innocent people, terrorize the whole community by its deadly results. This essay also points out the flaws in our health systems for example, are the health authorities is aware of the dangers of Avian flu are the health departments are serious about giving training to the members of health department to cope with this great threat of Avian flu, are they teaching local people how to cope with this danger? No I don't think that enough work is done to handle this great threat of Avian flu.

\section{References}

1. BBC NEWS. Q\&A: Bird flu. 2006.

2. CBC News. Avian influenza: FAQs. 2006.

3. Centres of Disease Control and Prevention. 2006. Avian influenza.

4. Centres of Disease Control and Prevention. 2005. Avian influenza.
5. Centres of Disease Control and Prevention. 2006a. Pandemic Flu.

6. Centres of Disease Control and Prevention.2006b.Pandemic influenza.

7. Centres of Disease Control and Prevention. 2006c. Avian influenza.

8. Department of Health. Bird flu and pandemic influenza: what are the risks?.2006

9. International SOS . Pandemic preparedness, Avian flu outbreak.2006.

10. Monto AS . Vaccines and anti viral drugs in pandemic preparedness. 2006.

11. Memphis Business journal. St. Jude technology to help stop spread of avian flu in France. 2006.

12. NHS . Explaining pandemic flu. 2002

13. Parry J. South East Asia sets up task force to tackle avian flu. 2004c.

14. Parry J. Mortality from avian flu is higher in previous outbreak. 2004a.

15. Scott Gottlieb. Research confirms human to human transmission of avian flu. 2005

16. U.S. Department of Health \& Human Services. HHS Buys More Antiviral Medication for the Strategic National Stockpile. 2006.

17. Wikipedia. Transmission and infection of H5N1. 2006.

18. World Health Organization. Responding to the avian influenza pandemic threat. 2005.

19. Centres of Disease Control and Prevention. 2004. Avian influenza.

20. Cook F, Buckley PA. Avian genetics A population and ecological approach. London: Academic press limited. 1989.

21. Duncan K. Hunting the 1918 Flu. Toronto: University of Toronto press. 2003

22. Peaker M. Avian physiology. London: Academic Press. 1975.

23. Pauling L. Vitamin C The common cold and the flu. SS: W.H. Freeman and company. 1976 